\title{
Liver transplantation for malignant hepatic tumors
}

Peter Neuhaus

Humboldt University of Berlin, Berlin, Germany 


\title{
Long term results of transplantation for HCC with or without cirrhosis: a 15 year-experience at Paul Brousse Hospital
}

\author{
René Adam, D. Azoulay, D. Castaing, D. Samuel, F. Saliba, C. Feray, H. Bismuth \\ Hôpital Paul Brousse, Villejuif, France
}

Hepatocellular carcinoma(HCC) still remains a controversial indication for liver transplantation (LT). The lack of organs has restricted the use of transplantation to patients with very limited intrahepatic tumor, and excluded those patients with a large $(>50 \mathrm{~mm})$ and/or a multinodular ( $>3$ nodules) HCC.The increasing use of alternatives to cadaveric full size organs such as split, domino or living related livers makes now possible to transplant some patients with HCC otherwise excluded from conventional indications of LT. However, the evaluation of long term results of LT for HCC is mandatory to define the subgroups of patients likely to benefit from such alternatives. From December 1984 to December 1999, 1482 LT were performed consecutively in 1304 patients. From this, 220 were done for HCC either in patients with underlying cirrhosis (195 cases) or without cirrhosis (25 cases). The main results according to the presence of underlying cirrhosis or not appears, as follows :

\begin{tabular}{|c|c|c|c|c|}
\hline & Cirrho & & Non & \\
\hline SURVIVAL $(\%)$ & 5 yrs & $10 \mathrm{yrs}$ & 5 yrs & $10 \mathrm{yrs}$ \\
\hline - Overall & 61 & 48 & 74 & 40 \\
\hline - Tumor Size & & & & \\
\hline$\leqq 30 \mathrm{~mm}$ & 69 & 62 & 67 & - \\
\hline $30-50 \mathrm{~mm}$ & 43 & 31 & 80 & 80 \\
\hline$>50 \mathrm{~mm}$ & 40 & 15 & 78 & 31 \\
\hline - $\underline{\text { Number of nod }}$ & & & & \\
\hline 1 & 72 & 70 & 100 & - \\
\hline $2-3$ & 58 & 35 & 50 & 25 \\
\hline$>3$ & 42 & 33 & 69 & 37 \\
\hline - Number + size & & & & \\
\hline 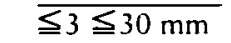 & 73 & 65 & 75 & - \\
\hline$\leqq 3>30 \mathrm{~mm}$ & 46 & 20 & 83 & 67 \\
\hline$>3 \leqq 30 \mathrm{~mm}$ & 51 & 43 & 60 & - \\
\hline$>3>30 \mathrm{~mm}$ & 35 & 25 & 74 & 37 \\
\hline - Portal invasion & & & & \\
\hline Yes & 25 & 0 & 51 & - \\
\hline No & 66 & 54 & 81 & 46 \\
\hline - Microvascular & & & & \\
\hline Yes & 47 & 33 & 59 & 25 \\
\hline No & 70 & 59 & 86 & - \\
\hline - Previous hepate & & & & \\
\hline Yes & 47 & 47 & 100 & 67 \\
\hline No & 60 & 48 & 68 & - \\
\hline RECURRENCE & & & & \\
\hline
\end{tabular}

\section{Comments:}

1- In spite of a higher incidence of recurrence related to more extensive tumors, long term survival of LT tends to be better for HCC without cirrhosis as compared to HCC with cirrhosis. This is particularly true for tumors larger than $30 \mathrm{~mm}$ and/or multinodular (>3).

2- Portal invasion by the tumor whichever is the branch, should remain an absolute contraindication to LT, in patients with cirrhosis.

3- By contrast, patients with underlying cirrhosis usually excluded from conventional indications of LT because of large $(>50 \mathrm{~mm}$ ) or multinodular $(>3)$ HCC could have appreciable long term survival $(40 \%$ and $42 \%$ at 5 years, respectively). These patients could benefit from alternative procedures of LT provided that no other risk factor related to the tumor is present. 


\title{
Living donor liver transplantation for malignant hepatic tumors
}

\author{
Koichi Tanaka \\ Department of Transplantation Immunology, \\ Kyoto University Graduate School of Medicine, Kyoto, Japan
}

[Background] Living donor liver transplantation had been started for patients with malignant hepatic tumors as a result of the spread of application to adult patients. However, there have been many controversies, including indication on the basis of tumor stage, immunosuppresive regimen, postoperative chemotherapy and preoperative ablation treatment.

[Methods] Between Fcbruary 1999 and January 2001, 30 cases with hepatocellular carcinoma received living donor liver transplantation. Criteria of indication consisted of no evidence of extrahepatic tumor and no evidence of macroscopic tumor involvement in portal or hpatic veins. Number or size of the tumor was not taken into consideration for criteria. Patients consisted of 22 males and 8 females with mean age of 49 y.o., ranging from 12 y.o. to 68 y.o. Underlying liver disease were hepatitis C (12), hepatitis B (11), hepatitis C and B (2), alcohol (1), citrullinemia (1) and unknown (3). There were one patients in Stage I, 3 in Stage II, 3 in Stage III and 22 in Stage IV-A by preoperative imaging examination. Immunosuppression consisted of tacrolimus monotherapy. For the assessment of the recurrence of tumor, AFP and PIVKA-II were checked monthly, and CT scan (abdomen, chest, brain) and bone scitigraphy were instituted every 3 months.

[Results] Eight recipients died of sepsis (3), peritonitis (2), pneumonia (1), cereberal bleeding (1) and asphyxia (1). Out of 22 surviving recipients, 2 had the recurrence of tumor (one with lung metastasis and tumor in the diaphragm, one with brain metastasis) during the mean follow-up period of 9 months.

[Conclusions] Further long-term follow-up is necessary to evaluate the efficacy of living donor liver transplantation for patients with hepatocellular carcinoma. 